\title{
EVOLUCIÓN DEMOGRÁFICA DEL ORIENTE CUBANO ENTRE 1700-1862
}

\section{DEMOGRAPHIC EVOLUTION OF EASTERN OF CUBA FROM 1700 TO 1862}

\section{Alexeis Díaz Pérez*}

RESUMEN

El siguiente artículo explica la evolución demográfica del Departamento Oriental cubano durante el siglo XVIII hasta el límite censal de 1862, facilitando la comprensión de las características más notables de la población oriental en este período. Para su realización fue imprescindible el análisis de diferente fuentes bibliográficas relacionadas con el tema; así como, los censos coloniales del período. El apoyo en la estadística matemática facilitó, desde este estudio cuantitativo, comprender cualitativamente el proceso.

PALABRAS CLAVE: CUBA * DEMOGRAFÍA * POBLACIÓN * MASCULINIDAD * MIGRACIÓN * ESCLAVITUD

\section{ABSTRACT}

The following article explains the demographic evolution of the Cuban Eastern Department population during the eighteenth century to the limit census of 1862 , facilitating the understanding of the most notable features of the eastern population in this period. For its realization was indispensable the analysis of different bibliographic sources related to the subject and the colonial census of the period. The support provided in mathematical statistics facilitated, from this quantitative study, a qualitative understanding of the process.

KEYWORDS: CUBA * DEMOGRAPHY * POPULATION * MASCULINITY * MIGRATION * SLAVERY

Universidad de Granma, Cuba adiazp@udg.co.cu 


\section{INTRODUCCIÓN}

El poblamiento está condicionado por la importancia económica de cada región. En dependencia de esto, así será el desarrollo en los patrones demográficos que irá adquiriendo, a la vez que las condiciones que posee regula el tipo de actividad a la que se va a dedicar $y$ estas marcan las características de la población. Las diferencias regionales durante la evolución colonial cubana estuvieron definidas "... por el predominio de las áreas de producción para la exportación, fundamentalmente azucareras, que incentiva el desarrollo de las ciudades comerciales, lo que produce el crecimiento de las zonas de producción para el consumo interno" (Barcia, García y Torres-Cuevas, 2002: 281).

El surgimiento del Departamento Oriental de Cuba en 1607, fue resultado del reconocimiento económico que adquirió la región para la Isla y la metrópoli española, fundamentalmente por lo atesorado en las minas de El Cobre de Santiago del Prado. Por otra parte, esta división sirvió para ejercer mayor control sobre la oligarquía bayamesa y su comercio de rescate (Portuondo, 1996: 27-28).

\section{DESARROLLO}

Durante el siglo XVIII, se dieron importantes segregaciones poblacionales en el Departamento, fundamentalmente desde Bayamo. Jiguaní, en 1700, aparece como pueblo de indios y Holguín, en 1752, surge como jurisdicción, sumándose a Bayamo (Santiago de Cuba) y Baracoa.

Llegando el siglo XVIII (1689), la población oriental era de 8232 habitantes en un promedio de 6,3 habitantes por familia. Esta representaba más de la mitad de la población de La Habana y el 25,2\% de la existente en la Isla.

GRÁFICO 1

CRECIMIENTO DE LA POBLACIÓN ORIENTAL SIGLO XVIII

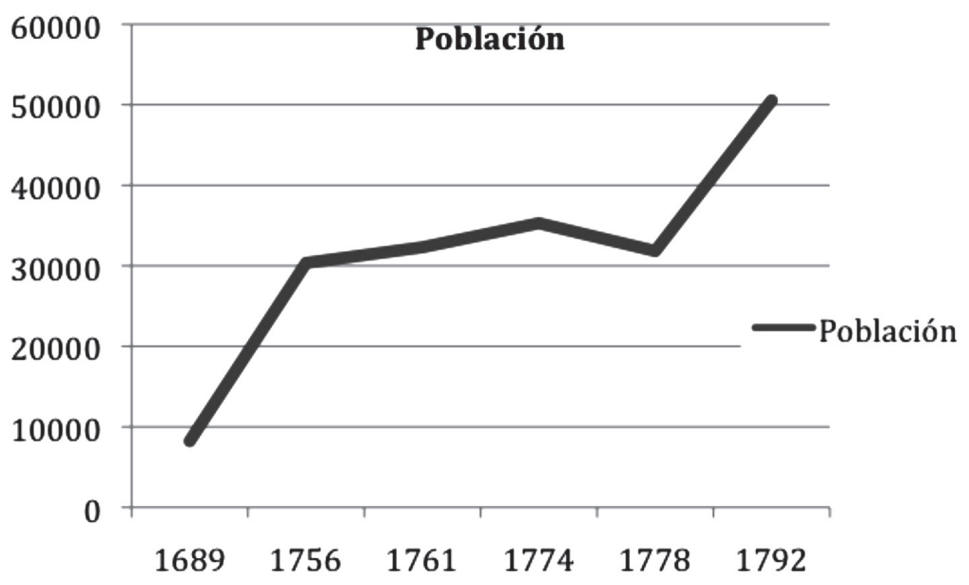

Fuente: Elaboración propia.

Para el año 1756, según datos aportados por Morell de Santa Cruz, entre sus cuatro jurisdicciones, su población se había multiplicado más de tres veces. Las 4836 familias, cifra que también se triplicó, albergaban 30356 personas. El fondo habitacional se componía de casas que eran fundamentalmente con techos de paja $(59,9 \%)$ y el resto de tejas.
La población que más se destacaba era la de Bayamo como fehaciente muestra del esplendor de la economía bayamesa, favorecida por su comercio y la existencia de grandes haciendas. En esta se encontraba el 44,4\% del total de la población del Oriente y era la segunda población de Cuba, con 12653 habitantes. Santiago de Cuba se encontraba 
situado como el cuarto centro poblacional de la Isla con 11793 habitantes.

Entre los años 1756-1778, el número de viviendas en el Departamento aumentó, reflejándose sustancialmente en las poblaciones de El Cobre y Holguín, no tanto así en Santiago de Cuba y Bayamo.

A mediados de este siglo, Nicolas Joseph de Ribera hizo refencia al desarrollo y estado de las poblaciones en el Oriente, al plantear: "Baracoa se ha atrasado mucho, Santiago, sin crecer ella, ha producido los pueblecitos del Cobre $y$ de Los Caneyes, $y$ a la ya ciudad de Holguín. San Salvador ha crecido algo, ha criado a Jiguaní y en alguna parte a Holguín" (De Ribera, 1975: 135).

El 3 de septiembre de 1761, el Capitán General de la Isla, Juan de Portocarrero, ordenó al Gobernador del Departamento Oriental,
Lorenzo de Madariaga, la ejecución de un censo de población. Este reveló una población total de 32303 habitantes (Censos de Población y Vivienda en Cuba, 1988). En todo un lustro, la cifra de crecimiento no alcanzó las 2000 nuevas almas, aportando una tasa anual de crecimiento de $1,24^{1}$. Se invierte además, la supremacía poblacional de Bayamo con respecto a Santiago de Cuba.

El censo apuntó a El Caney, El Cobre y Jiguaní como jurisdicciones independientes, categoría que no ostentaban en el período ${ }^{2}$.

Una de las preocupaciones de las autoridades del Departamento, era el hecho de que más de la mitad de la población de El Cobre era esclava, la cual devenida en sublevación podía poner en crisis al Gobierno Oriental. De la cifra de 1240 habitantes que presentaba, 735 eran esclavos que debieron estar empleados en las minas casi en su totalidad.

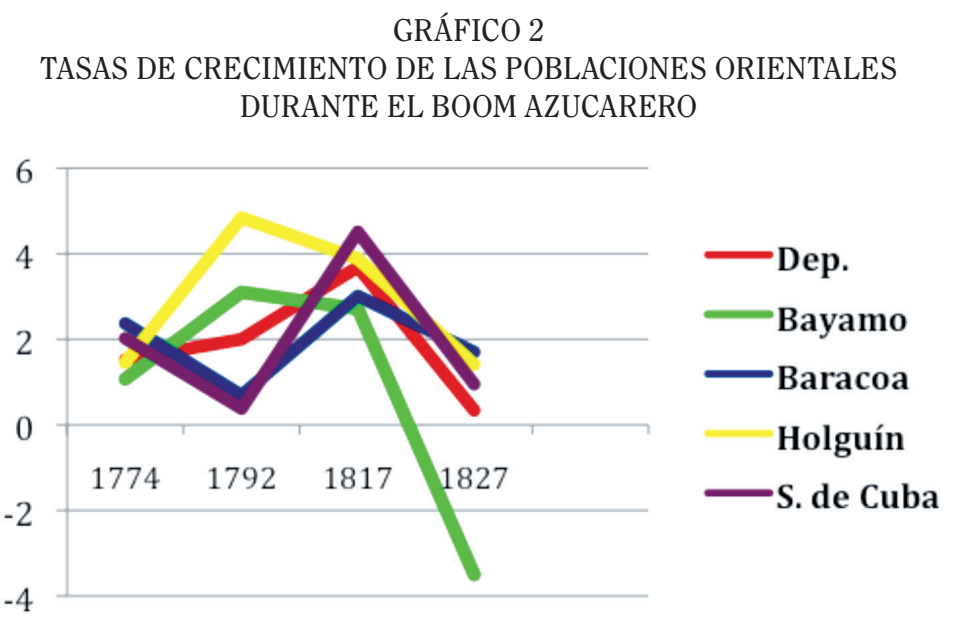

Fuente: Elaboración propia.

$\overline{1}$ Se ha utilizado la tasa de crecimiento geométrica, representando esta 1 por cada 100 habitantes.

Según Olga Portuondo Zúñiga, esto se debió a que el empadronamiento se realizó durante el período de dominación británica en La Habana y se consideraron estas localidades, por dicho motivo, como tenencias de gobiernos con el deliberado propósito de ejercer un mejor control en tiempo de guerra o bien, es una equivocación en la que se interpreta por jurisdicciones las que no eran sino partidos (Portuondo, 2003: 20-21). 
El promedio de habitantes por familia en el Departamento, era de 6,8 aproximadamente. Las poblaciones de Bayamo y Santiago de Cuba eran las que tenían el mayor promedio de habitantes por familias con 7,5. Los pueblos de El Cobre, Jiguaní y el Caney eran los de menor promedio de habitantes por familia, como tendencia que pudo haber marcado el desarrollo desigual entre las poblaciones catalogadas como pueblos y los de otras categorías.

En 1774, el Marqués de la Torre ordenó realizar el primer censo colonial oficial en Cuba. Este determinó que el Oriente de la Isla estaba habitado por 35286 personas, el 20,1\% de las existentes en Cuba. La población blanca era el 42,9\%, mientras la libre de color representaba un $33,0 \%$ y un $24,1 \%$ para la esclava.

Desde 1761, la población solo creció en unos 2982 habitantes, el 9,2\% de la población de 1761 , con una tasa de crecimiento de solo 0,68 , marcando un crecimiento poblacional todavía muy lento, si lo comparamos con posteriores momentos.

La población estaba notablemente marcada por la fuerte presencia de varones, situación muy paralela a la que experimentó la Isla de forma general durante el siglo XviII. Este sexo formó el $60,4 \%$ de toda la población oriental, por lo que el índice de masculinidad era bastante elevado. La proporción entre hombres y mujeres se comportaba con una presencia de 152 hombres cada 100 mujeres. La población esclava exhibió la mayor proporción de hombres con respecto a las mujeres, siendo de 193 hombres por cada 100 mujeres, seguido de la población blanca y la libre. El alto índice de masculinidad entre la población esclava, la baja tasa de fecundidad y natalidad que debió existir, unido a la alta tasa de mortalidad entre esta población, mayor que entre las demás, repercutieron de forma negativa en el crecimiento natural de la misma.

Entre las poblaciones de Baracoa y Santiago de Cuba, se encontraban los mayores índices de masculinidad. Dentro de la primera, su población libre de color manifestó el mayor índice de masculinidad, seguido de la población blanca, por cada 258 hombres libres de color habitaban 100 mujeres de igual condición. En el caso de Santiago de Cuba, fue la población esclava la que evidenció las mayores proporciones de hombres con respecto a mujeres, siendo de 253 hombres por cada 100 mujeres. Bayamo era la jurisdicción con el índice de masculinidad más bajo, su población tanto blanca como libre de color, ostentaban las menores relaciones de masculinidad entre la población de igual condición.

Por otra parte, la jurisdicción de Santiago de Cuba tenía más de la mitad de toda la población del Oriente, le siguió Bayamo con un poco más del $34 \%$. El hecho de que Santiago de Cuba haya equiparado tanta población del Oriente, respondió a la importancia económica de la jurisdicción y a la incapacidad de otras en atraer inmigración que las coloque en un lugar de reconocimiento dentro de las poblaciones cubanas. Holguín segregado de Bayamo en 1752, había logrado una acumulación poblacional que la hizo superar otros centros poblacionales importantes como Baracoa desde el año 1761. Su relación de masculinidad fue la más alta del Departamento para este año, lo que puede evidenciar que una parte considerable de esta debió emigrar hacia la jurisdicción.

El padrón de 1778 (Pérez, 1977: 5-16) expone para el Oriente cubano una población inferior que la censada en 1774, reflejando poca veracidad en las cifras recogidas, cuando la tendencia en la evolución poblacional oriental, por estos años, no fue hacia un decrecimiento de la población. Según el padrón, a excepción de la jurisdicción de Holguín, que tuvo una tasa de crecimiento de 5,31, en todas las restantes ocurre un decrecimiento en sus poblaciones. Este fenómeno, según las cifras, repercutió en el promedio de habitantes por casas, reduciéndose circunstancialmente en todas las poblaciones a excepción de Bayamo. Unos disminuyeron este promedio por el aumento del fondo habitacional y otros por la disminución de la población que albergaban.

El Departamento Oriental fue teniendo el crecimiento poblacional más bajo en Cuba. Esto se nota cuando el porcentaje medio de crecimiento de la Isla era de 27,6\% mientras que en el Oriente se mantenía un $16,9 \%$ de crecimiento poblacional. El Occidente denotó una progresión poblacional que respondía al propio fomento económico que se gestionaba y vivía, cuando este casi duplicó al del Departamento Oriental. 
CUADRO 1

COMPOSICIÓN POBLACIONAL SEGÚN CONDICIÓN SOCIAL Y COLOR DE PIEL 1788-1841

(PORCENTAJES)

\begin{tabular}{cccc}
\hline AÑO & BLANCO & LIBRE DE COLOR & ESCLAVO \\
\hline 1788 & 41,9 & 34,6 & 23,5 \\
1791 & 40 & 33 & 27 \\
1811 & 38 & 25 & 37 \\
1813 & 36,2 & 34,9 & 28,9 \\
1817 & 24,2 & 39,2 & 36,6 \\
1827 & 36,3 & 36,11 & 27,5 \\
1841 & 33,5 & 30,5 & 36 \\
\hline
\end{tabular}

Fuente: Elaboración propia.

La población oriental con 31831 habitantes se repartían entre un $46,7 \%$ de mujeres $y$ un $53,3 \%$ de hombres, con una proporción de 114 hombres por cada 100 mujeres, la masa esclava masculina era 1,5 veces más que la femenina. La población blanca era el 41,9\% aproximadamente, la población libre de color el 34,6\% y la esclava el 23,5\%.

Cuatro años más tarde (1792), la población era de 50532 habitantes, aumentando, con respecto a 1774, en 15246 pobladores, es decir, el 39,3\% de la población de 1774 .

Un análisis de la composición poblacional, atendiendo a su condición social y color de la piel, deja ver cierta correlación entre la población libre de color y la esclava en una etapa en que la evolución, hasta entonces bastante autóctona, del sistema esclavista en Cuba, acentuó estas características. La primera representó el $32,4 \%$ de la población del Departamento, mientras la esclava se reflejó en un $28,1 \%$ con 14190 esclavos, de los cuales el 50\% se concentraba en Bayamo, mientras que Holguín y Baracoa no llegaron a tener en conjunto un $7 \%$ de esta población, debido al tipo de actividad que desarrollaban, la cual no necesitó grandes montos de mano de obra como la demandada por la industria del azúcar.

Lo sucedido en Bayamo fue aparejado con el crecimiento del auge económico que vivió para esta época la jurisdicción. La concentración esclava y libre de color que presentó
Bayamo y Santiago de Cuba “... muestra la conjugación de los viejos elementos de la esclavitud patriarcal y la economía natural de las grandes zonas ganaderas con el desarrollo de la industria del azúcar que se producía en esos años" (La Rosa, 1991: 84).

La población que creció más aceleradamente fue Holguín, fundamentalmente, su masa esclava. Entre 1774 y 1792, su población casi se multiplicó por 2,4 veces. El crecimiento experimentado en 1792 significó, para esta jurisdicción, el 139,2\% de la población de 1774 , con una tasa de crecimiento de 4,84. Bayamo tuvo una tasa de crecimiento de 3,10, Baracoa 0,69 y Santiago de Cuba 0,38.

Se puede afirmar que entre estos años, de forma general, la población femenina fue la que más creció dentro del total poblacional oriental; su crecimiento entre 1774 y 1792 representó el $60,1 \%$ de la existente para este sexo en 1774 , con una tasa de crecimiento de 2,62 , mientras la tasa de crecimiento para los varones fue de 0,51.

El Oriente en su conjunto experimentó una tasa de crecimiento de 2,00, la masa esclava tuvo la tasa más alta de crecimiento con 2,84 , seguido por la población blanca y la libre de color.

Todo este fenómeno repercutió en la disminución del índice de masculinidad, pasando de la relación de 152 por cada 100 mujeres, en 1774 a 109 hombres por cada 100 mujeres. En todo el Oriente este indicador fluctuó entre 95 a 117 hombres por cada 100 mujeres. Baracoa tuvo las menores proporciones de hombres con respecto a mujeres, cuando en 1774 presentaba los mayores índices de masculinidad de todo el Departamento, lugar que fue ocupado por la población de Bayamo. Dentro de la masa esclava se exhibió una mayor relación de masculinidad; sin embargo, fue dentro de esta donde tuvo mayor connotación su reducción entre toda la población. Solo en la jurisdicción holguinera se distinguió un crecimiento, pasando de 99 hombres por cada 100 mujeres en 1774 a 113 hombres en 1792. Esta situación debió tener una substancial repercusión en los índices de fecundidad $y$ natalidad en la etapa. 
La masa esclava reflejó los mayores aumentos, sin dejar de tener en cuenta que los crecimientos dentro de la población femenina, tanto blanca como libre de color, fueron significativos.

Hasta este momento se puede entender que la evolución poblacional de la colonia cubana se desarrolló de forma bastante endógena, autóctona y bajo un crecimiento bastante natural. La última década del siglo xvIII, marcó el punto de partida de los cambios dentro de la composición poblacional oriental, donde las transformaciones dentro de la sociedad esclavista, trazaron la tendencia del movimiento poblacional.

En 1796, debido al desigual desarrollo de la región con respecto a Occidente, se decide llevar a cabo el fomento económico a través de la introducción de esclavos que permitieran satisfacer la demanda de mano de obra en la etapa. Además, a fines de siglo, la región se vio considerablemente favorecida por la llegada de inmigrantes procedentes de Haití como consecuencia de la Revolución, hasta entrando a la segunda mitad del siglo XIX, ocasionando un incremento poblacional de más de 44 mil habitantes. La llegada de los primeros refugiados a Santiago de Cuba ocurrió en 1793 y ya en 1808, según el padrón de ese año, constituían (con 7449 franceses), el $22 \%$ de la población de esta ciudad.

La población esclava y blanca fueron las que más crecieron, a contraparte de una evolución muy lenta en el crecimiento de la población libre en algunas zonas. En los lugares donde se dio un aumento se debe a "... la persistencia en ellas de la economía ganadera dentro de cuyo marco el proceso de emancipación legal era menos restringido" (La Rosa, 1991: 97).

Finalizando el siglo, se destacaron núcleos poblacionales como Manzanillo, que favorecido por las condiciones que le ofrecía el hinterland de Cauto, surge como ciudad. El interés por su fomento buscaba dotar a Bayamo de un puerto marítimo, evitar la penetración comercial y la amenaza militar enemiga (Marrero, 1978: 234).

Para 1810, la población del Oriente había crecido hasta 93304 habitantes, es decir, 42 772 más que en 1792, tanto por aumento de población libre como esclava.
En 1813, un padrón eclesiástico (Marrero, 1978: 234) identificó más de 125 mil habitantes en el Departamento. Coexistió una importante equivalencia entre la población en cuanto al color de la piel. La blanca representó el 36,2\% del total del Departamento, mientras la libre de color el $34,9 \%$ y un $28,9 \%$ para la esclava. En el Caney fue donde más se manifestó la existencia de población blanca, la cual representó el 93\%. El Cobre por su condición de zona minera, donde el trabajo era desarrollado fundamentalmente por gente de color, los blancos constituyeron solo el 2,2\% de su población, mientras la libre de color el 91,9\%. El padrón identificó a Bayamo como el Partido Eclesiástico de mayor población en 47644 habitantes, por delante de Santiago de Cuba, el cual contaba con 32494 habitantes ${ }^{3}$.

En 1817, la población fue de 127093 habitantes, de los cuales el 24,2\% eran blancos, el $39,2 \%$ libres de color y el $36,6 \%$ esclavos. La población que más creció en representatividad, dentro del total poblacional, con respecto a 1792 fue la esclava, aumentando en un 8,5\%, mientras la blanca disminuyó su representatividad.

El censo reflejó un momento importante en la evolución poblacional de todas las jurisdicciones orientales. La población experimentó un crecimiento apreciable, debido a que en este año se multiplicó por 2,5 con respecto a 1792 . La tasa de crecimiento fue de 3,69 , elevándose entre los libres de color y los esclavos. La población de Santiago de Cuba tuvo la mayor tasa de crecimiento, destacándose entre los esclavos y los libres de color. Bayamo tuvo el crecimiento más discreto, su tasa fue de 2,71, apreciándose un aumento importante entre la población blanca.

La población del Departamento tuvo un viraje considerable en cuanto a su composición atendiendo al color de la piel. Los blancos fueron desplazados de la supremacía numérica

3 Esto constituye un hecho poco probable por la importancia económica que ha adquirido Santiago de Cuba y que en todo este devenir, no manifestó rasgos de pérdida hegemónica frente a los demás centros poblacionales orientales, además de la tendencia ascendente en su crecimiento poblacional y su ratificación, por posteriores censos, siguió constituyendo la jurisdicción con mayor población en el Oriente cubano. 
que sustentaban. Su porcentaje con respecto al total poblacional del Departamento disminuyó en un 15,3\% comparado con 1792. Esto ocurrió en contraparte con el aumento de la población de color, tanto libre como esclava, que en su conjunto simbolizaban el $75,8 \%$ de la población, cuando en 1792 constituían el 60,5\%.

El crecimiento apreciado en estos años en el porcentaje de esclavos, tiene su explicación en la fuerte inmigración forzada que llega desde fines del siglo XviII hasta las primeras décadas del siglo XIX, como consecuencia de la decadencia que produjo en Haití la Revolución, en las producciones de azúcar y café. Esta colonia francesa fue cediendo lugar a Cuba dentro de su participación mundial como productora en estos ramos.

La trata negrera fue considerablemente en auge en el período, entrando los mayores porcentajes de esta masa en la etapa del boom azucarero cubano como demanda de mano de obra. Entre 1815/1822 entraron al
Oriente por el puerto de Santiago de Cuba, 25791 negros bozales.

Otra importante inmigración francesa se produce en el período procedente desde el año 1801 de Santo Domingo. Entre este año y 1802 llegaron a Cuba no menos de $30 \mathrm{mil}$ franceses que buscaban refugio en la región oriental, asentándose en Santiago de Cuba y en algunas zonas menos pobladas para encontrar tierras menos baratas para comprar o arrendar (Pichardo, 2006: 98-99).

En resumen, el crecimiento de la población esclava en estos años se dio por dos condiciones fundamentales: la creciente entrada de masa esclava a través de la trata negrera $y$ por el crecimiento natural que se experimentó en estos años por el auge azucarero. Sin embargo los libres de color tuvieron el aumento más destacado con respecto a la población de su condición de 1792. Fue entre los varones donde se hicieron notables los mayores aumentos.

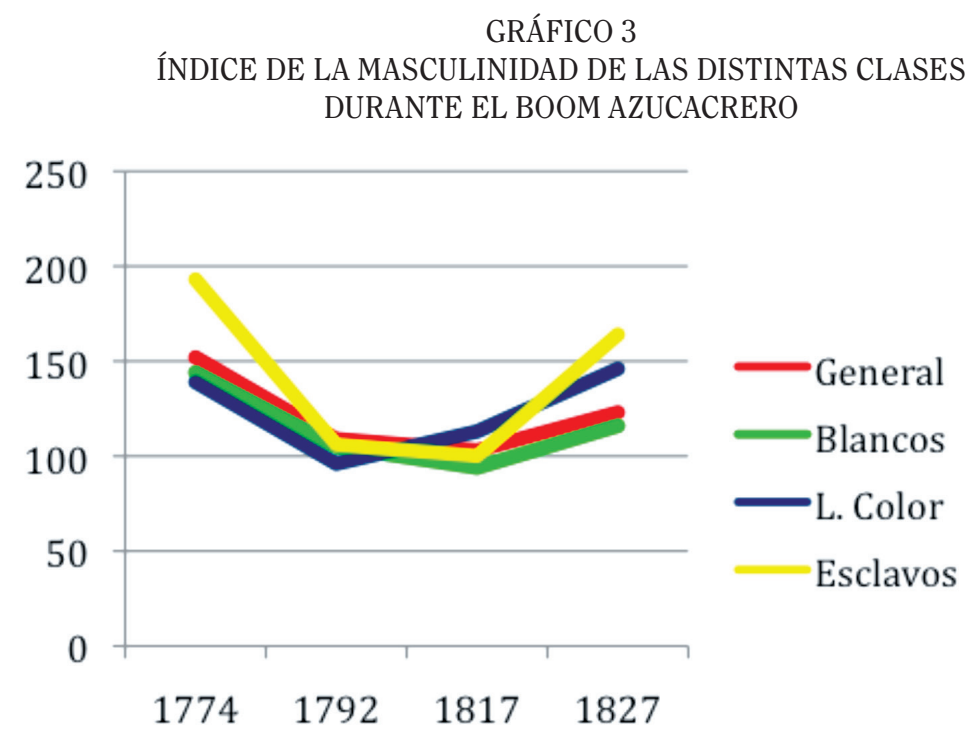

Fuente: Elaboración propia.

El porcentaje de población masculina dentro del total poblacional, después de haber descendido en más de un 11\% entre 1774-1778, llegó a ser el 52,3\% en 1792, por lo cual se mantendría bastante estable con respecto a años anteriores, siendo poco perceptible el crecimiento en la relación de masculinidad de 103 hombres por cada 100 mujeres. Bayamo mostraba el menor índice y Holguín el mayor. En el caso del primero, influyó la baja presencia de hombres esclavos con respecto a las mujeres de semejante condición, porque tanto en la población blanca, que 
presentó igual índice que en Holguín, como libre de color, tenía los mayores índices de masculinidad en todo el Oriente de Cuba dentro de la población de su condición respectiva.

Los libres de color, después de verse reducidos en este indicador entre 1774-1792, constituyeron los únicos capaces de aumentar en 25 años su relación de masculinidad. Tanto la población blanca como esclava, aunque en una medida poco apreciable, vieron disminuir la presencia de hombres ante la cantidad de mujeres. Dentro de la población blanca, solo en Holguín se notaron los aumentos en el índice de masculinidad, pasando de 99 hombres a 113 hombres por cada 100 mujeres.

La población esclava entrada en estos años no tuvo realmente una repercusión considerable en el índice de masculinidad, ya que no consiguió efectuar cambios que noten la huella de la inmigración forzada en la etapa en el referido aspecto, esto se puede observar cuando la población esclava femenina tuvo un incremento mucho mayor que el experimentado por la masculina con respecto al total de cada sexo correspondiente al año 1792, demostrándose que tanto el crecimiento natural de estos años, como la entrada de inmigración forzada, favorecieron más a la población esclava femenina que a la masculina.

Sin embargo, 10 años más tarde, en 1827, ocurrió un cambio circunstancial, en el que las consolidaciones de las producciones en estos años (1817-1827) necesitaron la fuerte presencia de mano de obra esclava. Los movimientos en el índice de masculinidad dentro de la masa esclava repercutieron significativamente en el ascenso del mismo, en la década, dentro del Departamento en su conjunto, el incremento de la población blanca masculina igualmente ejerció una destacada influencia en el cambio de dicho índice. Este consiguió elevarse hasta definir la presencia de 123 hombres por cada 100 mujeres.

Siguió constituyendo Holguín, la jurisdicción con mayor presencia de este sexo con relación a las mujeres; no obstante, el crecimiento del índice de masculinidad en Holguín como en Santiago de Cuba fue bastante semejante. La caída de este componente demográfico en Baracoa, jurisdicción que presentó el menor índice, constituyó un hecho interesante dentro del panorama oriental, en ella la presencia femenina formó más de dos veces la masculina, cuando por cada 42 hombres residían 100 mujeres.

A diferencia del período anterior (17921817), tanto la entrada de inmigración forzada y libre entre 1817-1827, como el propio crecimiento natural, condicionaron un incremento de la relación de masculinidad. A pesar de las restricciones para el desarrollo de la trata, que debían ponerse en práctica en 1820, no dejaron de entrar a Cuba esclavos por miles. Este hecho fue cardinal en el incremento del indicador en la etapa.

Dentro de la población esclava fueron más apreciables los cambios, pasando de 100 hombres por cada 100 mujeres, en 1817 aproximadamente, a 164 hombres por cada 100 mujeres en 1827, por lo que estos cambios debieron responder más al ingreso de inmigración forzada que a un crecimiento natural. Holguín fue la jurisdicción que más contribuyó en este sentido, al contener una población esclava masculina desproporcional con respecto a la femenina, donde por cada 301 hombres se apreció la presencia de 100 mujeres.

El impacto de este torrente migratorio no podía menos que alterar el patrón demográfico existente en Cuba. La naturaleza del movimiento respondía a los requerimientos de un sistema productivo sujeto a las oscilaciones del mercado internacional e inducía a estructurar las expediciones sobre la base de adultos en capacidad para laborar, preferentemente hombres, lo que posibilita la aplicación inmediata de brazos a la producción (...). Pero ello provocaba un desequilibrio sistemático en la estructura sexual de la población (García, 2003: 11).

El incremento también fue estimable, aunque con menor medida, dentro de la población libre, tanto blanca como de color. En la primera, el índice más bajo estuvo en Baracoa y el más alto en Santiago de Cuba, jurisdicción que también contenía la más baja relación de masculinidad de todo el Departamento dentro de su población libre de color. Dentro de esta última, fue también la jurisdicción holguinera la de mayor índice de masculinidad, con una relación de 177 hombres por cada 100 mujeres. 
GRÁFICO 4

TASA DE CRECIMIENTO DEL ORIENTE

1827-1862

Tasa

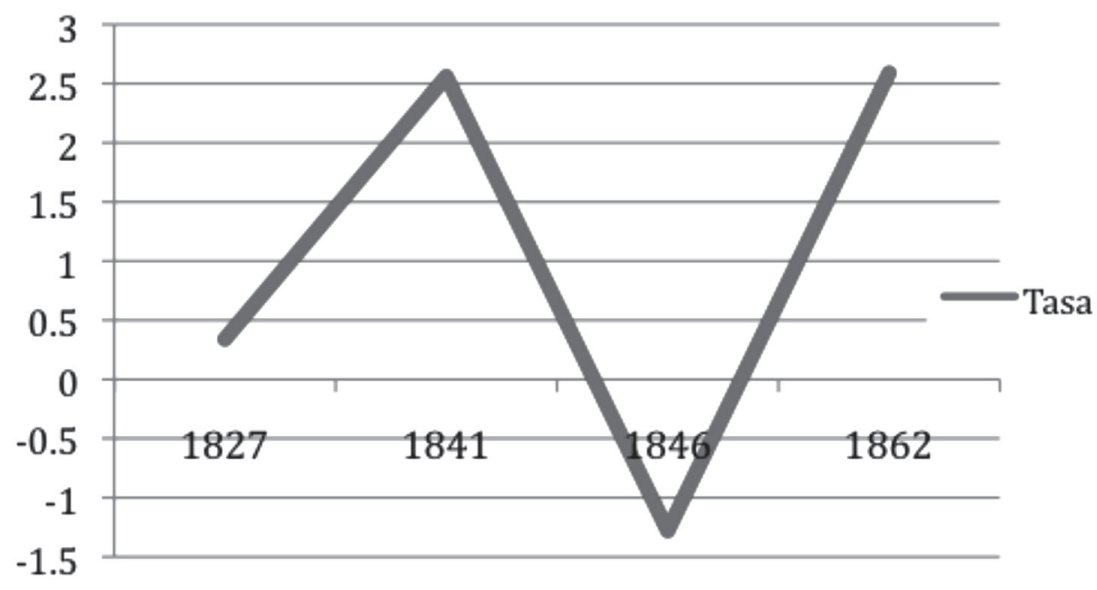

Fuente: Elaboración propia.

En 1827 (Censos de Población y Vivienda en Cuba, 1988), la población total del Oriente comprendía 131453 habitantes. La composición estamental de la sociedad estuvo bastante equilibrada. La población blanca constituyó casi la misma cantidad que la esclava con un $36,3 \%$ y $36,1 \%$ respectivamente, los libres de color representaban el 27,5\%. La población libre de color disminuyó, pero no significativamente, comparándola con 1817.

La proporción estamental oriental era de 36 blancos, 28 libres de color y 36 esclavos por cada 100 habitantes. Con anterioridad, el Oriente venía observando los cambios en proporciones poblacionales entre toda su población. Cabe destacar que en 1791, era de 40 blancos por 33 libres de color y 27 esclavos; en 1811 fue de 38, 25 y 37 respectivamente (Censos de Población y Vivienda en Cuba, 1988: 37).

Los habitantes por legua cuadrada fueron unos 106,9 como muestra del despoblamiento que aún subsistían en algunas zonas, un crecimiento poblacional que aunque bastante acelerado hasta la fecha, todavía no logra alcanzar a todas las zonas de la región, lo cual no permite a la vez ejercer una mayor explotación de áreas agrícolas y ganaderas. Las diferencias en la densidad poblacional en Cuba, entre sus distintas regiones, son considerables. En este propio año, la Isla presentaba un promedio de 201,5 habitantes por legua cuadrada, lo cual, comparándola con la densidad poblacional oriental demuestra que otras regiones se destacaban en este índice demográfico.

Entre 1817-1827, la población blanca aumentó de forma significativa con una tasa de crecimiento de 4,40, mientras la libre de color disminuyó en ambos sexos. La población esclava aunque aumenta, tuvo una tasa de solo 0,21, en la cual incidió exclusivamente Santiago de Cuba, ya que en todas las demás jurisdicciones disminuyó, solo el crecimiento ocurrió entre los varones, mientras la población esclava femenina se redujo; con esto surge la idea que este crecimiento en la masa esclava respondió más a las importaciones de estos que al crecimiento natural por estos años.

La menor población libre de color, nos da la medida del sustento esclavo para el desarrollo económico en la región, a pesar de que el crecimiento esclavo es más bajo que en el Centro y Occidente. De forma general, esta equivalencia entre el porcentaje de población blanca y la esclava se dio en toda la Isla. Sin 
embargo, dentro del propio Departamento, la situación no se reflejó de forma igual. Santiago de Cuba fue la jurisdicción que más incidió en este comportamiento, ya que el porcentaje de la población esclava era más de 2,4 veces el de la blanca, a pesar de que esta última tuvo la mayor tasa de crecimiento del Oriente cubano entre la población de igual condición, mientras que en las demás jurisdicciones, la población blanca excedió en más de 3 veces a la población esclava con excepción de Baracoa.

En la etapa se distinguía una evolución en el crecimiento poblacional muy lenta para algunas regiones, si se tiene en cuenta lo experimentado hasta entonces. Bayamo fue la población que más se vio afectada, teniendo un decrecimiento que representó el 29,5\% de la población existente en 1817. La población que más creció fue Baracoa, con una tasa de crecimiento de 1,70 aunque este solo constituyó el $18,5 \%$ de la población que albergaba en 1817. Le siguió Holguín y Santiago de Cuba.

Entrando la década del 40, se produjo un proceso de compensación poblacional entre la población blanca, libre de color y esclava. Por un lado, ha ocurrido una disminución de los blancos y esclavos a contraparte del aumento de los libres de color; estos últimos representaban el 30,5\% de la población, los esclavos que eran los de mayor número comprenden unos 65065 , el 36\% de la población del Oriente.

El censo terminado en 1841, demostró que el Oriente contenía una masa poblacional de 180256 habitantes. La población creció en 48803 habitantes, significando el 37,1\% de la población censada en 1827 , con una tasa de crecimiento de 2,56, destacándose las jurisdicciones de Baracoa y Holguín. El porcentaje de blancos del total de la población disminuyó en un $2,8 \%$, su tasa de crecimiento pasó de 4,40 en 1827 a 1,68, mientras acaeció un crecimiento entre los libres de color hasta alcanzar una tasa de 2,87, reflejándose en Baracoa su mayor tasa de crecimiento en la etapa.

La población esclava aumentó en 17566 , con una tasa de crecimiento de 2,25 , incidiendo en la jurisdicción de Holguín. Su porcentaje de representatividad, entre toda la población, solo varió en un $0,1 \%$, influenciado más por el aumento de población esclava femenina que masculina, lo que puede ser evidencia de que esta no fue tan importada como crecida de forma natural, a pesar de que a partir de 1825 “... los plantadores intentaron compensar las desproporciones de sexos en las expediciones..." (García, 2003: 12).

A pesar de esta situación, se dieron importantes progresos urbanos. Algunas poblaciones comenzaron a ser contempladas como ciudad, otras como villas y crecieron el número de poblaciones en categoría de pueblo. Los núcleos urbanos más importantes siguieron siendo Santiago de Cuba y Bayamo. La población urbana de Santiago de Cuba era 3,3 veces más que la de Bayamo. La ciudad de Holguín fue el tercer centro urbano y le seguían en orden Manzanillo, Baracoa y Tunas.

Para estos años, aunque existió una semejanza entre la cantidad de hombres $y$ mujeres que habitaban en el Departamento, los hombres excedían a estas, representando el $54,3 \%$ del total poblacional. Fue entre los libres de color que se dio un equilibrio de la población por sexo, donde cada una representó la mitad de su condición. Entre los blancos y esclavos, los varones tuvieron un mayor porcentaje, apreciándose las mayores diferencias entre la población esclava. 
GRÁFICO 5

ÍNDICE DE MASCULINIDAD DE LAS POBLACIONES ORIENTALES 1827-1862

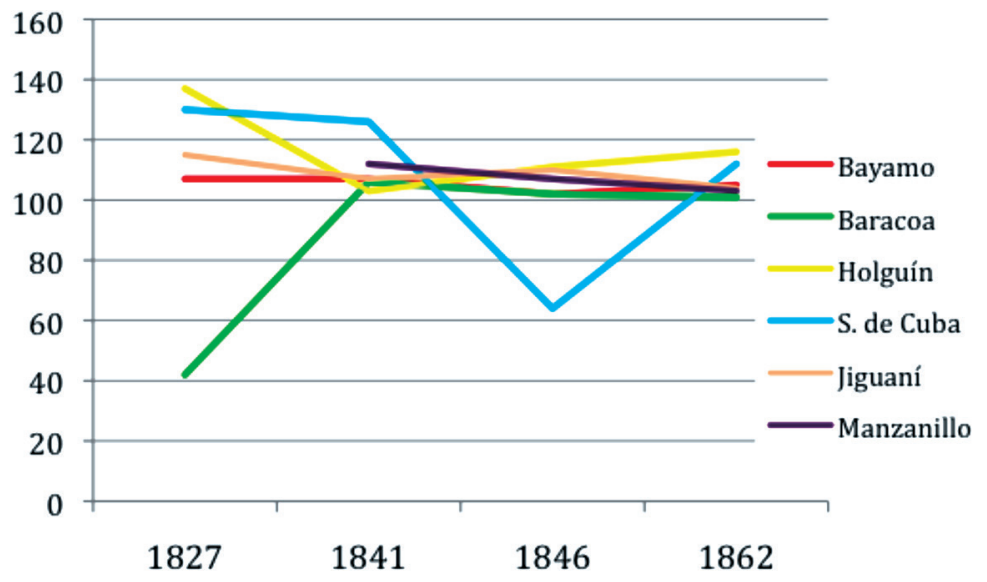

Fuente: Elaboración propia.

A partir de 1827, la tendencia en la evolución del índice de masculinidad fue hacia un decrecimiento. Este pasó de 123 hombres por cada 100 mujeres a 119 hombres por cada 100 mujeres. La población de El Cobre, por la fuerte presencia de esclavos en las minas, resultó ser la de mayor índice. Lo más impresionante se observó dentro de la jurisdicción holguinera, la cual en 1827 presentaba la mayor relación de masculinidad; no obstante, evolucionó demográficamente de tal forma que en catorce años pasó a ser la de menor presencia de hombres con respecto a las mujeres, llegando a ser de 103 hombres por cada 100 mujeres. Sin embargo, contenía el mayor índice de masculinidad dentro de la población libre. Santiago de Cuba siguió teniendo la mayor relación de masculinidad dentro de la población blanca en 1827, a la vez que Jiguaní mostró la menor. Dentro de la masa esclava de Guisa estuvieron los mayores índices de masculinidad de toda la población esclava del Departamento.

La disminución del índice de masculinidad, aunque fue apreciable entre toda la población, dentro de la libre de color ocurrieron los mayores cambios, esta manifestó un equilibrio bastante estable entre la presencia de hombres en proporción a las mujeres, por cada 100 hombres constaban también aproximadamente 100 mujeres.

Los habitantes en poblaciones constituyeron el 30,2\%, mientras el resto vivió en los campos, reflejo de que la vida económica se desarrollaba en zonas rurales por el peso que tenía el azúcar y el café, en la economía departamental. En los alrededores de los cafetales, que llegan a la cifra de 661, vivía el $23,4 \%$ de la población del campo, exhibiendo la mayor concentración poblacional como zona de producción. La población que vivía en el campo se encontraba distribuida fundamentalmente entre las fincas, sitios de crías y estancias. Los ingenios albergan un 9,1\% de la población rural.

Según datos del censo de $1846^{4}$, el Oriente estuvo habitado por 169082 personas (Censos de Población y Vivienda en Cuba, 1988: 99), disminuyendo en 11174 personas. Solo en Jiguaní y Manzanillo se reflejó un crecimiento,

4 El censo tuvo fuertes dificultades, por las omisiones de población, fundamentalmente esclava, hechas por sus dueños, lo que ha llevado a algunos estudiosos a omitir el mismo dentro de los censos coloniales. Este en ocasiones brinda tendencias, en otras no se ajusta a la dinámica del desarrollo general que experimentó la colonia cubana, pero como todos los demás, es muestra del panorama vivido en Cuba durante la etapa. 
con una tasa de 3,10 y 2,40 respectivamente, expresándose cierta inestabilidad en el crecimiento poblacional entrando la cuarta década del siglo XIX con respecto a otras zonas del país y que sirvió para marcar fuertes diferencias regionales, además de que la población del Departamento solo simbolizaba el $24,2 \%$ del total de la Isla. Dentro de la población blanca, solo se manifestó el crecimiento poblacional con una tasa anual de crecimiento de 1,99, destacándose en este sentido Bayamo. La población libre de color consiguió crecer en las poblaciones de Manzanillo, Jiguaní y Santiago de Cuba con una tasa de crecimiento de 4,61, 3,43 y 1,55 , respectivamente.

Los resultados del censo revelaron síntomas de la lenta evolución de la sociedad esclavista en Cuba, que para esta década ve escasear la mano de obra esclava, la cual disminuye considerablemente con respecto a 1841. Los 48962 esclavos cuantificados, constituyeron el $29 \%$ de toda la población, disminuyendo en $7 \%$ con respecto al anterior censo colonial. La población libre de color no sufre importantes cambios, solo se redujo en un poco más del $1 \%$ del total poblacional del departamento.

Es importante precisar que el crecimiento natural y la llegada de inmigración blanca a Cuba, propiciaron que esta población fuese ocupando un importante lugar dentro de la composición poblacional oriental, aumentando de un $33,5 \%$ en 1841 a un $39,4 \%$ para 1846 .

En cinco años, el campo siguió acogiendo la mayor parte de la población; además, durante este lustro la tendencia fue hacia la obtención de una mayor concentración poblacional con respecto a los pueblos, como generalidad dentro de la sociedad cubana, marcada por la economía agrícola. La población en el campo pasó de un $69,8 \%$ en 1841 a un $71,6 \%$ en 1846, reservando solo el $28,4 \%$ de sus habitantes para residir en poblaciones. En el campo, el $5,7 \%$ vivía cerca de los ingenios, el $23,8 \%$ en cafetales, el $31,8 \%$ en vegas y el $17,5 \%$ en otros tipos de fincas. Guantánamo tenía el porcentaje más alto de población rural con un $86,4 \%$ y Santiago de Cuba el más bajo con $65,6 \%$.

Las diferencias en la evolución poblacional entre los dos centros de población más importantes del Oriente, Santiago de Cuba y Bayamo, fueron notables. La primera, creció tanto con respecto a Bayamo, que la superaba en más de tres veces; aunque en 1861 se produce un aumento debido al progreso socioeconómico de esta última ciudad.

Entrando la década del 60, en la región Oriental “... la población se concentraba, principalmente, en los núcleos urbanos o en las unidades productivas, como los ingenios, cafetales y minas, pues aún predominaban las áreas incultas y las grandes extensiones de pastos naturales" (La Rosa, 1991: 148). Fue entre estos tipos de unidades productivas del Oriente cubano, donde se manifestaron, aún con menor densidad poblacional con respecto a los centros urbanos, los mayores porcentajes de habitantes totales de cada territorio.

En 1862, según el censo que terminó el 1ro. de junio, la población alcanzó las 255919 personas. Esto significó el 18,8\% del total de la Isla, disminuyendo en un $5,4 \%$ su representatividad dentro del total poblacional insular con respecto a 1846 . La composición poblacional estuvo dada por un $79,7 \%$ de libres, la masa esclava era el $19,9 \%$ y un $0,3 \%$ y $0,1 \%$ para los colonos y emancipados respectivamente (Barcia, García y Torres-Cuevas, 2002: 408-410). Fue notable la presencia de colonos llegados a Cuba como parte del fomento de la inmigración blanca gestionada y demandada para estos años de crisis de la sociedad esclavista. En 1858, se produjo la primera llegada de inmigrantes asiáticos a Santiago de Cuba traídos por Cayetano Milá en el barco vapor "Cuba" (Bacardí, 1913: 231).

El porcentaje de esclavos con respecto a 1846 disminuyó, pero este hecho solo se manifestó dentro de los hombres, en el caso de las mujeres aumentó. La proporción de blancos aumentó y la de color disminuyó como manifestación de la decadencia en la trata de esclavos y el fomento de inmigración blanca, tanto colonos asiáticos como yucatecos. Sin embargo, fue trascendente el aumento de los libres de color, favorecido también por el propio proceso de emancipación legal que se dio durante este período, teniendo una tasa de crecimiento anual de 2,88 , mientras la población blanca tuvo una tasa de crecimiento de 
2,59, hechos que repercutieron también en la disminución del porcentaje de esclavos dentro del total poblacional oriental, su tasa de crecimiento solo fue de 0,24 , que con excepción de Guantánamo y Manzanillo, en todas las demás poblaciones disminuye.

La población esclava representaba el $13,8 \%$ de la existente en la colonia, cifra mucho más baja que su representatividad dentro de la población general del Departamento. Santiago de Cuba tenía la mayor cantidad de esclavos, aunque solo representó el 8,43\% del total insular y tuvo para este año un decrecimiento, mientras que Guantánamo por su condición de cafetalera necesitó por mucho tiempo el empleo de esta mano de obra, de ahí que sostenga unos 8638 esclavos para la época.

Es apreciable el aumento en la tasa de crecimiento poblacional en Holguín que después de haber decrecido, llega a ser 5,48 en 1846. La mayor influencia en este sentido la ejerció la tasa de crecimiento entre los libres de color, pasando de un decrecimiento de esta a una tasa anual de 6,57, la más alta del Oriente. Sin embargo, dentro de la jurisdicción de Guantánamo, se hicieron latentes las mayores tasas de crecimiento entre su población blanca $(9,46)$ $y$ esclava $(4,22)$ con respecto a la población de igual condición del Departamento Oriental, influyendo en que esta jurisdicción atesorara la mayor tasa de crecimiento del Oriente cubano.

El índice de masculinidad siguió la misma tendencia que tomaría a partir de 1817. Los cambios dados en las jurisdicciones de Santiago de Cuba es un elemento que puede cuestionar los propios datos, ya que en seis años, la relación de masculinidad descendió hasta 64 hombres por cada 100 mujeres, cuando en 1841 era de 126 hombres por cada 100 mujeres, la segunda más alta del Departamento.

La población blanca de Bayamo tuvo el menor índice de masculinidad dentro de toda esta condición en el Departamento, mientras que en Guantánamo se observaban las mayores proporciones de hombres sobre mujeres dentro de toda la población de esta condición. Esta propia jurisdicción contenía el mayor índice de masculinidad entre los libres de color y el menor entre la masa esclava, hecho que influyó en que esta población tuviera las mayores relaciones de masculinidad del Oriente cubano. Jiguaní contenía dentro de la masa esclava, las mayores cifras en este componente demográfico.

De forma general, la relación de masculinidad, a pesar de haberse reducido, solo se exhibió entre la población blanca y esclava; disminuyendo la presencia masculina en 4 unidades, para cada una, por cada 100 mujeres con respecto a 1841, ya que dentro de la población libre de color se mantuvo estable.

La relación de masculinidad descendió hasta 110 hombres por cada 100 mujeres. Aunque dentro de la población blanca esta aumenta con respecto a 1846 , se mantendría notablemente inmutable dentro de los libres de color y notablemente disminuido dentro de los esclavos. En todo este panorama influyó el incremento de la inmigración blanca, por lo general masculina, el crecimiento natural con prevalencia a crecer más la población blanca y libre de color que la esclava, el proceso de emancipación legal y la disminución de la trata con respecto a décadas anteriores.

La jurisdicción con mayor índice de masculinidad siguió siendo Guantánamo, con mayor influencia, en este sentido, de su población blanca. La población libre de Holguín tuvo la mayor presencia masculina comparada con otras poblaciones, similar a la esclava en el caso de Jiguaní.

El declive poblacional que experimentó Bayamo y que se manifestó en la década del 60 del siglo XIX, motivada por la pérdida de la importancia económica de la jurisdicción, es notoria a través del lugar que fueron ocupando otros centros poblacionales como es el caso de Manzanillo. En 1862, esta ciudad tenía una población de 5643 habitantes, mientras que Bayamo surgida tres siglos antes ve decaer su masa poblacional y solo albergaba unas 6119 personas (Marrero, 1978: 234).

La estructura social del Oriente estuvo sustentada fundamentalmente en los labradores y los esclavos, como fuerza social y económica actuante en el período.

Cabe destacar que para 1792, la población blanca era la de mayor porcentaje dentro del Departamento. Como consecuencia de la 
alta introducción de masa esclava, para el año 1817 , se ve notablemente reducida a un $24,2 \%$, mientras la libre de color y esclava aumentaron. Aunque aumentó bruscamente para 1827, la población blanca vuelve a disminuir en 1841, a partir de aquí fue de forma ascendente.

La población libre de color también experimentó este proceso ascendente entre los años 1841-1862, mientras la esclava fue descendiendo, tomando en 1862 el porcentaje más bajo durante todo el siglo XIX.

El índice de masculinidad experimentó un crecimiento desde fines del siglo XviII, sin embargo, a partir de fines de la década del 20 del siglo xix, sufrió una caída en dicho indicador demográfico, manteniéndose un poco estable hasta entrada la década de 1860 .

\section{CONCLUSIONES}

La evolución poblacional que experimentó la región Oriental durante el siglo XVIII hasta 1862 , respondió a la dinámica que se desenvolvió en la Isla, donde múltiples fenómenos internacionales tuvieron para el Oriente cubano, la misma repercusión en los patrones demográficos que en el resto de la Isla. Sin embargo, determinados factores regionales influyeron en las características de esta, como es el caso de las actividades que van a prevalecer en la zona Oriental.

A pesar de los importantes movimientos en la composición poblacional en la región Oriental, como ocurrió durante el boom azucarero, no significaron fenómenos que pudieron situarlos en una balanza competitiva con otras regiones de la colonia como la parte Occidental de la Isla.

El período del boom azucarero, con la llegada de inmigraciones, tanto europea como forzada, el lento progreso económico que se percibe luego en el Departamento Oriental como parte del inicio de la crisis de la sociedad esclavista cubana, constituyeron importantes estímulos que llevaron a los cambios acelerados $y$ luego lentos, dentro de las poblaciones orientales en el período.

Estos fenómenos generaron una fuerte presencia de población de color entre fines del siglo XVIII y las primeras décadas del siglo XIX, pasando la década del 40 hacia una sociedad donde el incremento de la inmigración blanca $y$ la disminución de la trata, cambiaron la composición poblacional hacia una marcada presencia de población blanca, aunque los índices de masculinidad siguieron siendo significativos.

\section{BIBLIOGRAFÍA}

Almodóvar Muñoz, Carmen (1986). Antología crítica de la Historiografía cubana. Época Colonial. 2da edición. La Habana: Editorial Félix Varela, 2006.

Archivo Histórico Provincial de Granma. "Portuondo Zúñiga, Olga: el Departamento Oriental visto a través de los padrones (1756-1766)". Expediente 141. Archivo Vertical. Legajo 7.

Bacardí Moreau, Emilio. Crónicas de Santiago de Cuba. Tomo I, II y III. Edición Especial Limitada. Barcelona: Tipografía de B. Bauza, 1913.

Barcia, María del Carmen; García, Gloria y Torres-Cuevas, Eduardo (1994). Historia de Cuba. La Colonia: evolución socioeconómica y formación nacional de los orígenes hasta 1867. Tomo I. Segunda Parte. 2da edición. La Habana: Editorial Félix Varela, 2002.

Censos de Población y Vivienda en Cuba. Estimaciones. Empadronamientos y censos de población de la época colonial y la primera intervención norteamericana. Tomo I, volumen 1 y 2 . Instituto de Investigaciones Estadísticas, diciembre de 1988, año 30 de la Revolución.

Cepero Bonilla, Raúl. Escritos históricos. La Habana: Editorial de Ciencias Sociales, 1989.

Colectivo de Autores. La esclavitud en Cuba. 1ra edición. La Habana: Instituto de Ciencias Históricas-Editorial Academia, 1986.

De Humboldt, Alejandro. Cuadro estadístico de la Isla de Cuba. 1825-1829. La Habana: Imprenta "Marón", 1965.

De Humboldt, Alejandro. Ensayo político sobre la Isla de Cuba. La Habana: Fundación Fernando Ortiz, 1998. 
De Ribera, Nicolás Joseph. Descripción de la Isla de Cuba. La Habana: Editorial de Ciencias Sociales, 1975.

De Santa Cruz, Morell. Visita Eclesiástica. La Habana: Editorial de Ciencias Sociales, 1985.

García, Gloria. La esclavitud desde la esclavitud. 2da edición. La Habana: Editorial de Ciencias Sociales, 2003.

Guerra, Ramiro. Manual de Historia de Cuba. 6ta edición. La Habana: Editorial Pueblo y Educación, 1985.

La Rosa Corzo, Gabino. Los palenques del Oriente de Cuba. Resistencia y acoso. 1ra edición. La Habana: Editorial Academia, 1991.

Le Riverend, Julio. Historia económica de Cuba. 2da edición. La Habana: Editorial Pueblo y Educación, 1971.

Marrero, Levi. "El siglo XviII. Azúcar, ilustración y conciencia (1763-1868) (I)”. Economía y Sociedad. Tomo Ix. Madrid: Editorial Playor SA, 1978.

Pérez de la Riva, Juan. "Presentación de un censo ignorado: el Padrón General de 1778". Revista de la Biblioteca Nacional José Martí 3. Septiembre-diciembre, 1977: 5-16.
Pérez de la Riva, Juan. El monto total de la inmigración forzada en el siglo XIX. 1ra edición. Ciudad de la Habana: Editorial Ciencias Sociales, 1979.

Pichardo Viñals, Hortensia. Temas históricos del Oriente cubano. La Habana: Editorial de Ciencias Sociales, 2006.

Pinos Santos, Oscar. Cuba, historia y economía. La Habana: Editorial de Ciencias Sociales, 1984.

Portuondo Zúñiga, Olga. Santiago de Cuba desde su fundación hasta la Guerra de los Diez Años. 1ra edición. Santiago de Cuba: Editorial Oriente, 1996.

Portuondo Zúñiga, Olga. Entre esclavos y libres de Cuba colonial. 1ra edición. Santiago de Cuba: Editorial Oriente, 2003.

Ramos Piñol, Oscar. El primer censo de la población de Cuba colonial. La Habana: Editorial Estadística, 1990.

Venegas Fornias, Carlos. Cuba y sus pueblos: censos y mapas de los siglos XVIII y XIX. Centro de Investigación y Desarrollo de la Cultura Cubana Juan Marinello, 2002.

Fecha de ingreso: 14/06/2012 Fecha de aprobación: 22/02/2013 
